\title{
RhoE controls myoblast alignment prior fusion through RhoA and ROCK
}

\author{
M Fortier ${ }^{1}$, F Comunale ${ }^{1}$, J Kucharczak ${ }^{1}$, A Blangy ${ }^{1}$, S Charrasse ${ }^{1}$ and C Gauthier-Rouvière ${ }^{*, 1}$
}

Differentiation of skeletal myoblasts into multinucleated myotubes is a multi-step process orchestrated by several signaling pathways. The Rho small G protein family plays critical roles both during myogenesis induction and myoblast fusion. We report here that in $\mathrm{C} 2 \mathrm{C} 12$ myoblasts, expression of RhoE, an atypical member of this family, increases until the onset of myoblast fusion before resuming its basal level once fusion has occurred. We show that RhoE accumulates in elongated, aligned myoblasts prior to fusion and that its expression is also increased during injury-induced skeletal muscle regeneration. Moreover, although RhoE is not required for myogenesis induction, it is essential for myoblast elongation and alignment before fusion and for M-cadherin expression and accumulation at the cell-cell contact sites. Myoblasts lacking RhoE present with defective p190RhoGAP activation and RhoA inhibition at the onset of myoblast fusion. RhoE interacts also with the RhoA effector Rhoassociated kinase (ROCK)I whose activity must be downregulated to allow myoblast fusion. Consistently, we show that pharmacological inactivation of RhoA or ROCK restores myoblast fusion in RhoE-deficient myoblasts. RhoE physiological upregulation before myoblast fusion is responsible for the decrease in RhoA and ROCKI activities, which are required for the fusion process. Therefore, we conclude that RhoE is an essential regulator of myoblast fusion.

Cell Death and Differentiation (2008) 15, 1221-1231; doi:10.1038/cdd.2008.34; published online 28 March 2008

The Rho family of Ras-like GTPases consists of signaling molecules involved in cytoskeleton remodeling, membrane recycling and gene transcription. Rho GTPases play essential roles in controlling cell adhesion, migration, cell proliferation and differentiation ${ }^{1}$ and particularly in skeletal myogenesis. ${ }^{2-10}$ The Rho subfamily contains 21 members clustered in four subgroups that comprise RhoA, RhoB and RhoC; Rnd1, Rnd2 and Rnd3/RhoE; RhoBTB1, RhoBTB2 and RhoBTB3; and Rac1, Rac2, Rac3, Cdc42, TC10, TCL, RhoG, Chp1 and Chp2/Wrch-1. The remaining proteins (RhoD, Rif and RhoH/TTF) delineate distinct stems in the phylogenetic tree. Rho GTPases act as molecular switches that convert extracellular signals into multiple cellular effects by cycling between an inactive GDP-bound and an active GTP-bound state. Their activation is controlled by guanine nucleotide-exchange factors (GEFs), whereas their inactivation is promoted by GTPase-activating proteins (GAPs). The members of the Rnd subfamily (Rnd1, Rnd2 and Rnd3 also called RhoE) and RhoH are an exceptions, as they are always bound to GTP, are not regulated by GEFs and GAPs and have very low, if any, intrinsic GTPase activity. ${ }^{11-14}$ Although 21 members are known in mammals, only few of them have been studied extensively. In skeletal muscle cells, the role of RhoA, Rac1 and TC10 has been analyzed. RhoA and Rac1 act both in myogenesis induction and myoblast fusion. RhoA positively regulates MyoD expression and skeletal muscle cell differentiation, as it is required for serum response factormediated activation of several muscle-specific gene promoters. ${ }^{2}$ Conversely, Rac1 inhibits myogenesis induction by preventing myoblast withdrawal from the cell cycle. ${ }^{3,15}$ Later on, during the skeletal muscle differentiation program and in contrast to its inhibitory role in myogenesis induction, Rac1 signaling is involved in myoblast fusion in Drosophila. ${ }^{4-7,16}$ Recently, we demonstrated that Rac1 activity is required for myotube formation, ${ }^{17}$ whereas RhoA activity must be downregulated during this process, ${ }^{8,9,18}$ as that of the Rhoassociated kinase (ROCK)I, an effector of RhoA. ${ }^{9,18}$ In analyzing the potential mechanisms that might be involved in downregulation of RhoA and ROCKI during myoblast fusion, we focused on RhoE. Indeed, RhoE is ubiquitously expressed and is believed to be a RhoA and ROCKI antagonist. ${ }^{19}$ RhoE was isolated in a two-hybrid screen that used p190RhoGAP as bait $^{11}$ and shown to stimulate p190RhoGAP-mediated GAP activity toward RhoA. ${ }^{20}$ Moreover, RhoE binds and inhibits ROCKI activity. ${ }^{21}$ Here we show that RhoE is involved in mammalian myoblast fusion in the myogenic cell line $\mathrm{C} 2 \mathrm{C} 12$. We demonstrate that $\mathrm{RhoE}$ expression increases until the onset of fusion, whereas at the same time the activity of RhoA decreases. Finally, we also show that the knockdown of $R h o E$ by RNA interference impairs downregulation of RhoA and ROCK activities normally observed before myoblast fusion and thus inhibits myotube formation.

\footnotetext{
${ }^{1}$ Universités Montpellier 2 et 1, CRBM, CNRS, UMR 5237, IFR 1221919 Route de Mende, 34293 Montpellier, France

${ }^{*}$ Corresponding author: C Gauthier-Rouviere, CRBM, CNRS, UMR 5237, IFR 122, Universités Montpellier 2 et 1, 1919 Route de Mende, Montpellier 34293 , France. Tel: + 33467613 355; Fax: + 33467521 559; E-mail: cecile.gauthier@crbm.cnrs.fr

Keywords: RhoE; myoblast fusion; RhoA; ROCK

Abbreviations: DM, differentiation medium; GAP, GTPase-activating protein; GEF, guanine nucleotide-exchange factor; GM, growth medium; MLCK, myosin light chain kinase; ROCK, Rho-associated kinase

Received 20.6.07; revised 26.2.08; accepted 28.2.08; Edited by G Cossu; published online 28.3.08
} 


\section{Results}

Variation of RhoE expression during skeletal muscle cell differentiation. RhoE is ubiquitously expressed and its mRNA has also been detected in skeletal muscle contrary to Rnd1 and Rnd2, the other members of this Rho family subgroup that were not observed in this tissue. ${ }^{13}$ To analyze their mRNA expression in C2C12 myoblasts, we performed real-time quantitative $\mathrm{PCR}$ amplification using specific primer sets. $^{22}$ RhoE mRNA was found in $\mathrm{C} 2 \mathrm{C} 12$ myoblasts and its level was close to that of RhoA and Rac1, which are both involved in the regulation of myogenesis (Figure 1).

Rnd proteins are always bound to GTP and variations in their expression might be used to control their activities. We next analyzed RhoE mRNA and protein levels in $\mathrm{C} 2 \mathrm{C} 12$ myoblasts induced to differentiate by replacing the growth medium (GM) with the appropriate differentiation medium (DM). At different time points after the shift to DM, cell extracts were prepared to analyze both mRNA and protein levels. The fusion process was concomitantly followed by morphological analysis of myoblast differentiation and appearance of multinucleated myoblasts. We could thus determine that fusion started between 30 and $42 \mathrm{~h}$ after DM addition (Figure $2 \mathrm{~A}$ ). As shown in Figure 2B, RhoE mRNA gradually increased until the onset of fusion and then suddenly decreased. These variations in RhoE mRNA levels coincided with those of RhoE protein (Figure $2 \mathrm{C}$ ). These results show that RhoE is upregulated at the onset of myoblast fusion.

To analyze whether RhoE participates in skeletal muscle differentiation in vivo, we examined its expression in a mouse model of muscle regeneration. Skeletal muscle injury was induced by injection of notexin or cardiotoxin in tibialis anterior (TA) muscles. Regeneration was monitored by histological analysis (Figure 2Da) and M-cadherin expression (Figure 2Db) 4 days after injection. RT-PCR amplification showed that the expression of $R h o E$ and M-cadherin were concomitantly upregulated during regeneration.

RhoE is required for myotube formation. To gain further insight into the role of RhoE in myogenesis, we knocked down RhoE expression. We generated stable $\mathrm{C} 2 \mathrm{C} 12$ cell

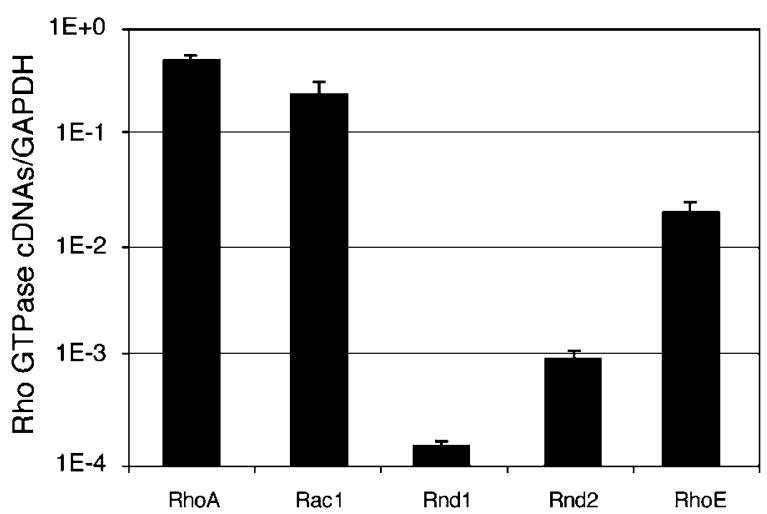

Figure 1 RhoE expression in $\mathrm{C} 2 \mathrm{C} 12$ myoblasts. RhoA, Rac1 and RhoE cDNA levels relative to GAPDH were determined by quantitative RT-PCR amplification. The graph is representative of three independent experiments lines in which RhoE was silenced by small hairpin RNAs (C2C12 RhoE shRNA). We monitored the efficiency of gene silencing by western blot analysis in five different clones (Figure 3A). In all clones, RhoE protein levels relative to that of Tubulin were decreased by $50-80 \%$ (Figure 3B). Parental and $\mathrm{C} 2 \mathrm{C} 12$ myoblasts expressing Firefly Luciferase-specific shRNA (C2C12 Luci shRNA) were used as controls. Parental, C2C12 RhoE shRNA and Luci shRNA myoblasts were grown to $80 \%$ confluency and shifted to DM for 4 days. Whereas numerous myotubes were observed in the parental and control C2C12 Luci shRNA cells (Figure 3Ca-b), only few myotubes were visible in RhoE shRNA clone C2 (Figure $3 \mathrm{Cc}$ ). Similar results were obtained with all five RhoE shRNA clones, demonstrating that these effects were not due to clonal variations (data not shown). Quantification of the fusion index clearly illustrates that the knockdown of $R$ hoE impairs myotube formation (Figure 3D). We next examined, by western blot analysis, whether RhoE silencing affected the expression of Myogenin and Troponin T, two musclespecific proteins (Figure 3E). Their expression was similar in parental, control C2C12 Luci shRNA and C2C12 RhoE shRNA myoblasts, suggesting that induction of these genes does not require $\mathrm{RhoE}$.

These data indicate that RhoE is necessary for myotube formation while being dispensable for myogenesis induction.

RhoE controls myoblast elongation and alignment before fusion. We next analyzed RhoE distribution during myogenesis by indirect immunohistochemistry using a specific, affinity-purified antibody against RhoE (Figure 4A). In proliferating $\mathrm{C} 2 \mathrm{C} 12$ myoblasts, RhoE had a predominantly perinuclear localization (a) as in fibroblasts. ${ }^{23}$ After DM addition, RhoE staining increased in myoblasts that were elongating and aligning before fusion (b). Once fusion occurred, it decreased to a level similar to that of proliferating cells (d). Neither the pre-immune serum (data not shown) nor the affinity-purified antibody pre-incubated with the immune peptide (c) revealed such a staining.

We then precisely analyzed the morphological modifications occurring during the myogenesis of parental and $\mathrm{C} 2 \mathrm{C} 12$ RhoE shRNA myoblasts using phase contrast and scanning electron microscopy (SEM) (Figure 4B and C). During myogenesis, $\mathrm{C} 2 \mathrm{C} 12$ myoblasts elongate and align before fusion (Figures $4 \mathrm{Ba}-\mathrm{c}$ and $5 \mathrm{Ca}-\mathrm{d}$ )..$^{24,25}$ In contrast, RhoE knockdown prevented elongation and alignment of myoblasts that remained flat (Figures $4 \mathrm{Bd}-\mathrm{f}$ and $5 \mathrm{Ce}-\mathrm{h}$ ). Higher magnification analysis by SEM of the dorsal membrane revealed the presence of extensive microvilli and lamellipodia in parental $\mathrm{C} 2 \mathrm{C} 12$ at the onset of myoblast fusion (Figure 4Cd), which were absent in C2C12 RhoE shRNA myoblasts (Figure 4Ch).

$\mathrm{M}$-cadherin, a member of the $\mathrm{Ca}^{2+}$-dependent cell-cell adhesion molecule family, is involved in myoblast alignment and fusion. ${ }^{8,26}$ We thus examined M-cadherin expression and localization in C2C12 RhoE shRNA myoblasts (Figure 4Da). M-cadherin was monitored at different times after DM addition. In C2C12 RhoE shRNA myoblasts cultured in GM, the amount of M-cadherin was similar to that of the parental C2C12 cells. M-cadherin expression decreased in $\mathrm{C} 2 \mathrm{C} 12$ RhoE shRNA myoblasts grown in DM compared to parental 
A

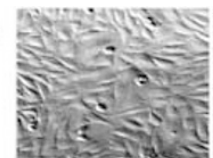

GM

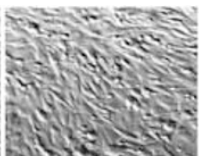

$30 \mathrm{~h}$

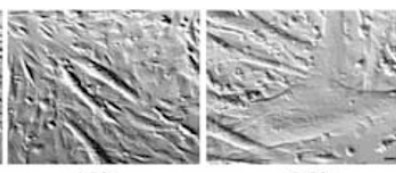

$42 \mathrm{~h}$

$96 \mathrm{~h}$

B

Myotube formation
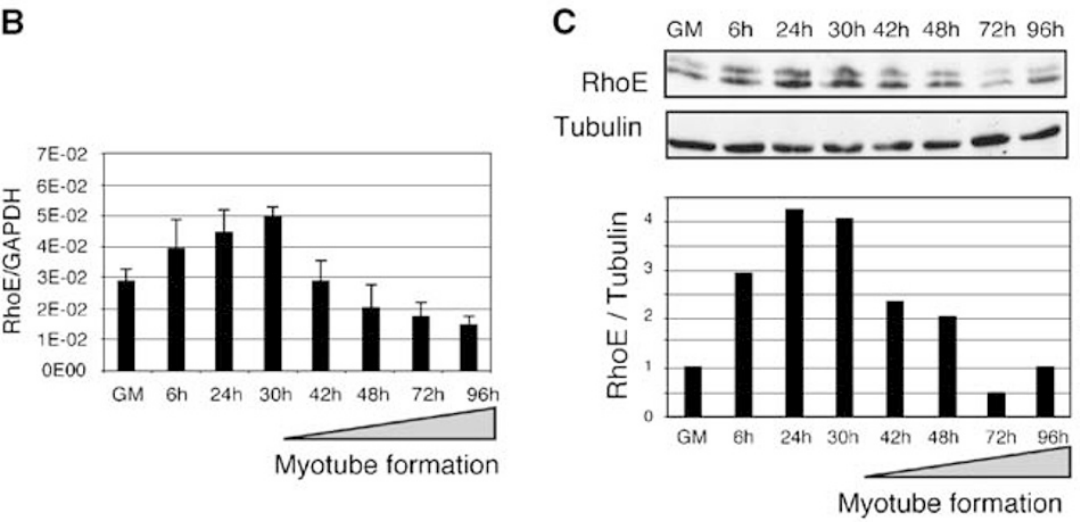

D a

b
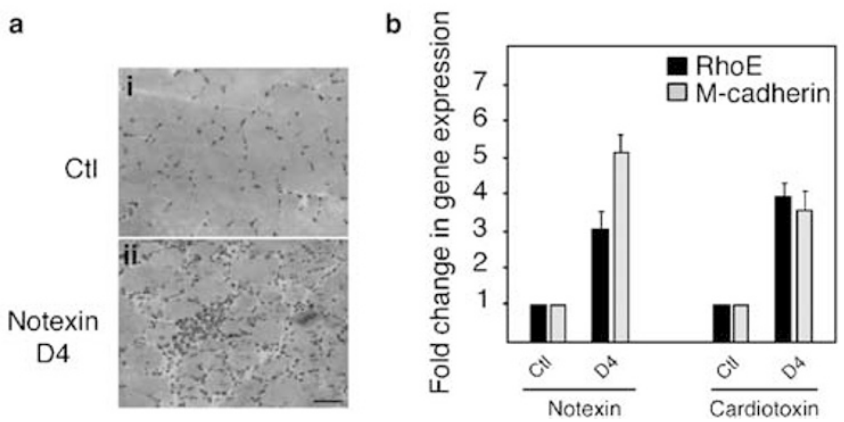

Figure 2 RhoE expression during $\mathrm{C} 2 \mathrm{C} 12$ myoblasts differentiation. C2C12 myoblasts grown in GM or in DM for the indicated times were simultaneously processed for the analysis of the fusion process $(\mathbf{A})$ and for mRNA $(\mathbf{B})$ and protein extracts preparation $(\mathbf{C})$. (A) Phase contrast images of myoblasts and myotubes during the differentiation process. Bar: $20 \mu \mathrm{m}$. (B) The graph shows the RhoE cDNA levels relative to the GAPDH level in the same sample at the indicated periods, determined by quantitative RT-PCR amplification. Data are the mean \pm S.E.M. of five independent experiments. (C) Protein extracts (50 $\mu \mathrm{g} / \mathrm{well})$ from C2C12 myoblasts, collected at the indicated times, were immunoblotted for the assessment of RhoE and $\alpha$-Tubulin expression. The histogram represents the quantification of RhoE normalized for the amount of $\alpha$-Tubulin. The histogram shows the quantification of the two bands but similar variations have been observed for each of these bands (data not snown). (D) (a) Serial sections from control (i) and injured TA muscles 4 days after notexin injection (ii) were stained with hematoxylin and eosin. Bar: $100 \mu \mathrm{m}$. (b) Expression of M-cadherin and RhoE determined by quantitative RT-PCR amplification after normalization to GAPDH 4 days after notexin or cardiotoxin injection. The graph is representative of three independent experiments

C2C12 myoblasts (Figure 4Da). In contrast, N-cadherin expression was comparable in C2C12 RhoE shRNA and parental $\mathrm{C} 2 \mathrm{C} 12$ myoblasts (data not shown). We then analyzed M-cadherin localization by immunocytochemistry (Figure 4Db). In control elongated and aligned $\mathrm{C} 2 \mathrm{C} 12$ myoblasts, M-cadherin accumulated at myoblast-myotube and myotube-myotube contacts (i and ii), but not in $\mathrm{C} 2 \mathrm{C} 12$ RhoE shRNA cells (iii and iv).

Altogether, these data show that RhoE accumulates in elongated, aligned myoblasts prior to fusion and that its silencing impairs myoblast elongation and alignment and decreases M-cadherin expression and accumulation at intercellular contacts.

RhoE controls the downregulation of RhoA activity at the onset of myoblast fusion. RhoE antagonizes RhoA activity, ${ }^{12,20}$ which is highly regulated during myogenesis. Indeed, RhoA is activated and required for myogenesis induction, but its activity must be downregulated to allow myotube formation. ${ }^{2,8,9,27}$ First, we measured RhoA activity in $\mathrm{C} 2 \mathrm{C} 12$ myoblasts from the same kinetic as the one described in Figure 2. Figure 5 shows the crude data $(A)$ and RhoA activity normalized to the amount of total protein (B). As previously reported, we observed an increase in RhoAGTP level at the beginning of myogenesis. Then, RhoA-GTP content decreased and reached a minimum after 2 days in DM (i.e., at the onset of fusion) (Figure 5B). As the samples used for the experiments described in Figures 2 and 5 were similar, we reported the amount of RhoE protein onto the same graph (Figure 5B) and showed that the maximum RhoE expression correlated to the lowest RhoA activity. We next measured RhoA activity in C2C12 RhoE shRNA 
myoblasts. In control $\mathrm{C} 2 \mathrm{C} 12$ cells, we observed a decrease in RhoA activity $30 \mathrm{~h}$ after DM addition, that is, at the onset of fusion. Conversely, in C2C12 RhoE shRNA cells, we could not detect a decrease in RhoA activity at $30 \mathrm{~h}$ after DM addition (Figure $5 \mathrm{C}$ ) or at later time points. This suggests that RhoE-dependent pathways are not involved in the control of RhoA activation during myogenesis induction and that they are implicated in RhoA activity's downregulation at the onset of myoblast fusion. To further demonstrate that RhoA activity was maintained in $\mathrm{C} 2 \mathrm{C} 12$ RhoE shRNA myoblasts, we used the organization of the F-actin cytoskeleton as a functional readout (Figure 5D). After 3 days in DM, parental myoblasts showed a decrease in the number of stress fibers (Figure $5 \mathrm{Da}$ and $\mathrm{b}$ ), particularly in myotubes, as previously reported. ${ }^{25}$ In contrast, C2C12 RhoE shRNA myoblasts still presented with a higher amount of stress fibers, a hallmark of RhoA activation (Figure 5Dc and d).

To confirm that myoblast fusion inhibition after RhoE silencing was dependent on continuous RhoA activation, we treated $\mathrm{C} 2 \mathrm{C} 12$ RhoE shRNA myoblasts with the cell-perme-
A

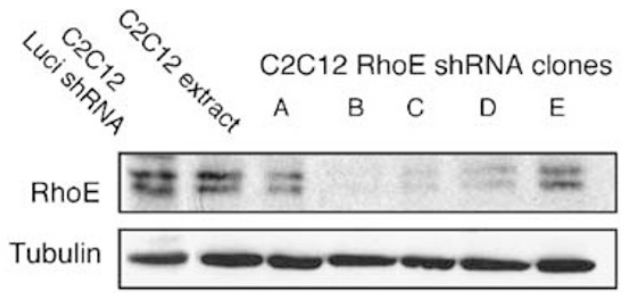

B

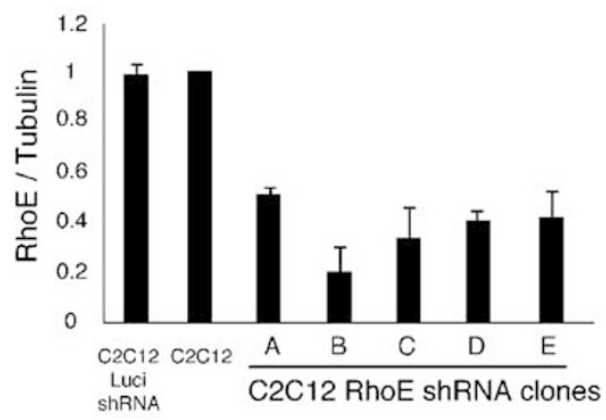

E

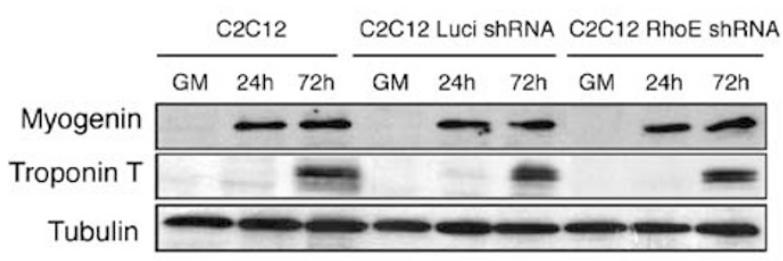

C

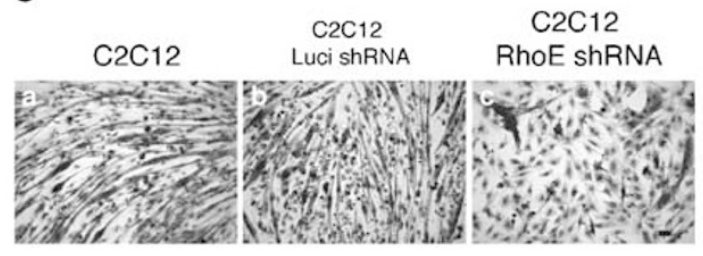

D
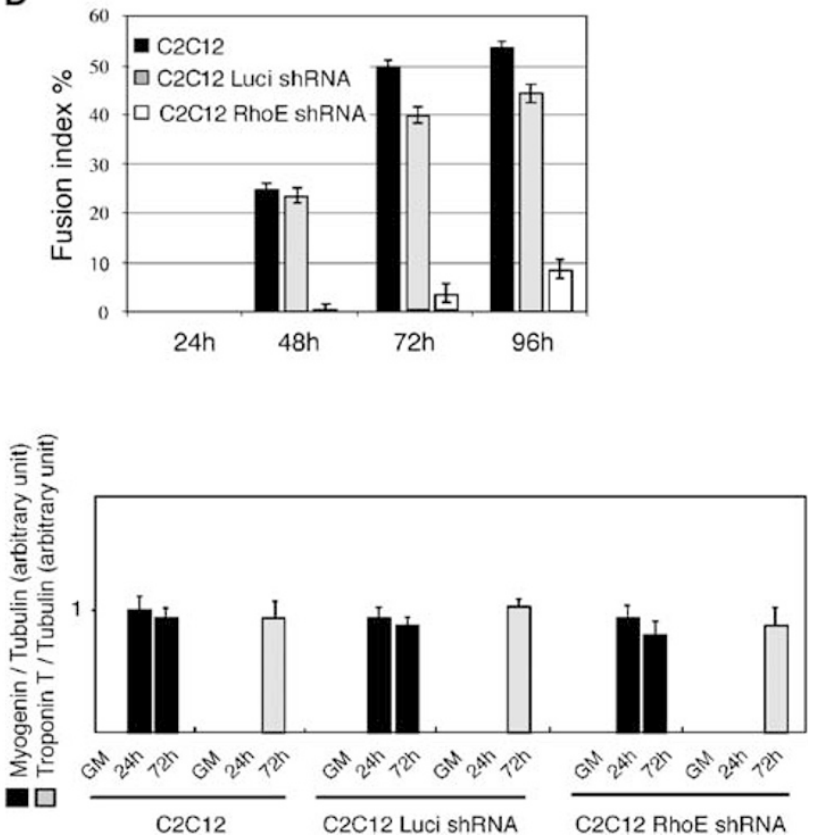

Figure 3 Inhibition of RhoE expression by RNA interference prevents myotube formation. (A) Luciferase and RhoE shRNAs were delivered in C2C12 myoblasts by retroviral infection. Cells were selected and clones of resistant cells were analyzed for the expression of RhoE and $\alpha$-Tubulin by immunoblot analysis. (B) The histogram represents the quantification of RhoE normalized for the amount of $\alpha$-Tubulin in the different clones. Data are the mean \pm S.E.M. of three independent experiments. The histogram shows the quantification of the two bands but similar variations have been observed for each of these bands (data not snown). (C) May-Grünwald Giesma stain of parental (a), control C2C12 Luci shRNA (b) and C2C12 RhoE shRNA myoblasts (clone B, c) after 4 days in DM. Bar, $50 \mu$ m. (D) The histogram represents the fusion index calculated at the indicated times after DM addition for parental, control C2C12 LucishRNA and C2C12 RhoE shRNA myoblasts (clone B). Data are the mean \pm S.E.M. of three independent experiments: at least 7000 nuclei were counted per experiment. (E) Cell lysates (20 $\mu \mathrm{g} /$ well) of parental, control C2C12 Luci shRNA and C2C12 RhoE shRNA myoblasts cultured in GM or DM for the indicated periods were assessed by western blot analysis for the expression of Myogenin, Troponin T and $\alpha$-Tubulin. The histogram represents the quantification of Myogenin (black) and Troponin T (gray) normalized for the amount of $\alpha$-Tubulin from four independent experiments

Figure 4 Inhibition of RhoE expression by RNA interference prevents myoblast elongation and alignment. (A) RhoE localization analyzed by indirect immunofluorescence in proliferating $\mathrm{C} 2 \mathrm{C} 12$ myoblasts (a) or after 30 (b) or $96 \mathrm{~h}$ (d) in DM. Staining obtained with the affinity-purified RhoE antibody pre-incubated with the immune peptide (c). Bar: $10 \mu \mathrm{m}$. (B) Phase contrast images of parental (a-c) and C2C12 RhoE shRNA myoblasts (d-f) cultured in GM or DM for the indicated periods. Bar: $10 \mu \mathrm{m}$. (C) Scanning electron micrographs of parental and C2C12 RhoE shRNA myoblasts cultured in GM or DM for the indicated periods. Bar: $10 \mu \mathrm{m}$. (D) (a) Protein extracts (20 $\mu \mathrm{g} /$ well) from parental and C2C12 RhoE shRNA myoblasts collected at the indicated periods were immunoblotted for M-cadherin and $\alpha$-Tubulin expression. The histogram represents the quantification of M-cadherin normalized for the amount of $\alpha$-Tubulin in three independent experiments. (b) M-cadherin localization in parental (i, ii) and C2C12 RhoE shRNA (iii, iv) myoblasts analyzed by indirect immunofluorescence. Shown are deconvolved images of M-cadherin (green) and DNA staining (blue). Bar: $10 \mu \mathrm{m}$ 
able exoenzyme C3, which inactivates RhoA. ${ }^{28}$ As RhoA activity is required for myogenesis induction, ${ }^{2,3}$ C3 transferase was added $6 \mathrm{~h}$ after induction of differentiation. As shown in Figure $6 \mathrm{~A}$, RhoA inhibition partially rescued inhibition of myoblast fusion caused by RhoE silencing. Quantification of the fusion index 3 days after DM addition revealed a four-fold increase in myoblast fusion in C2C12 RhoE shRNA myoblasts treated with $\mathrm{C} 3$ transferase, as compared to the untreated

A
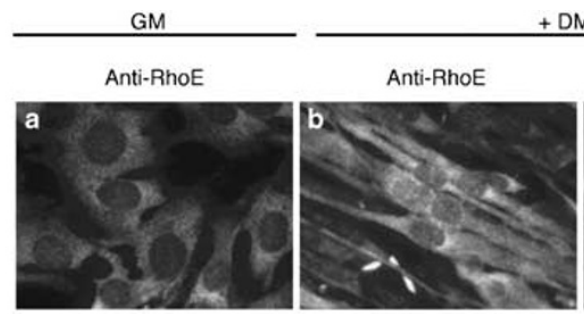

DM 30h

Anti-RhoE +

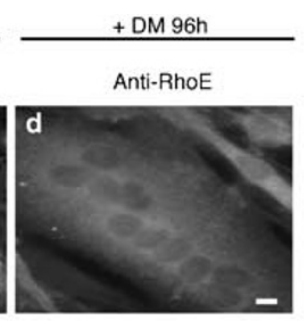

B

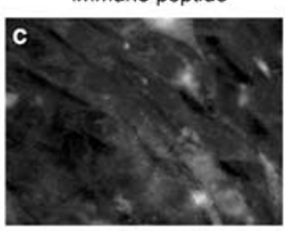

$+\mathrm{DM}$

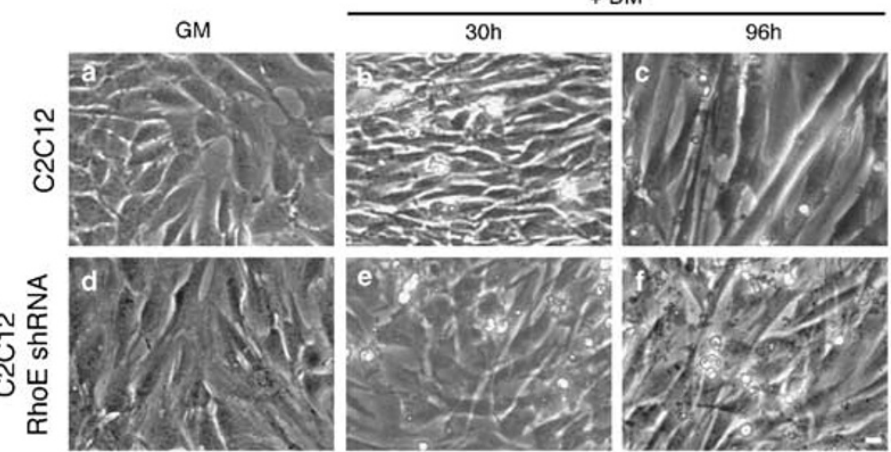

C
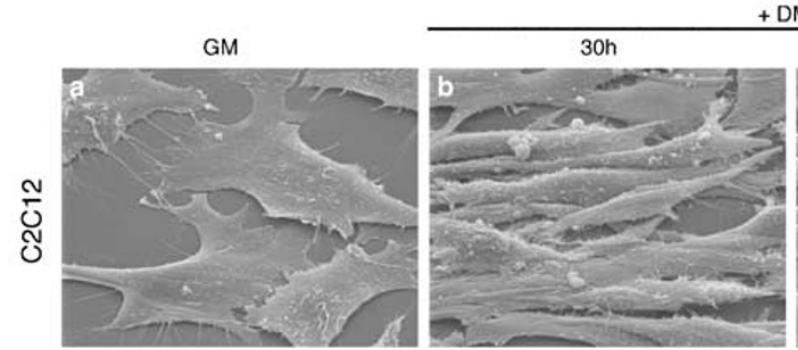

DM
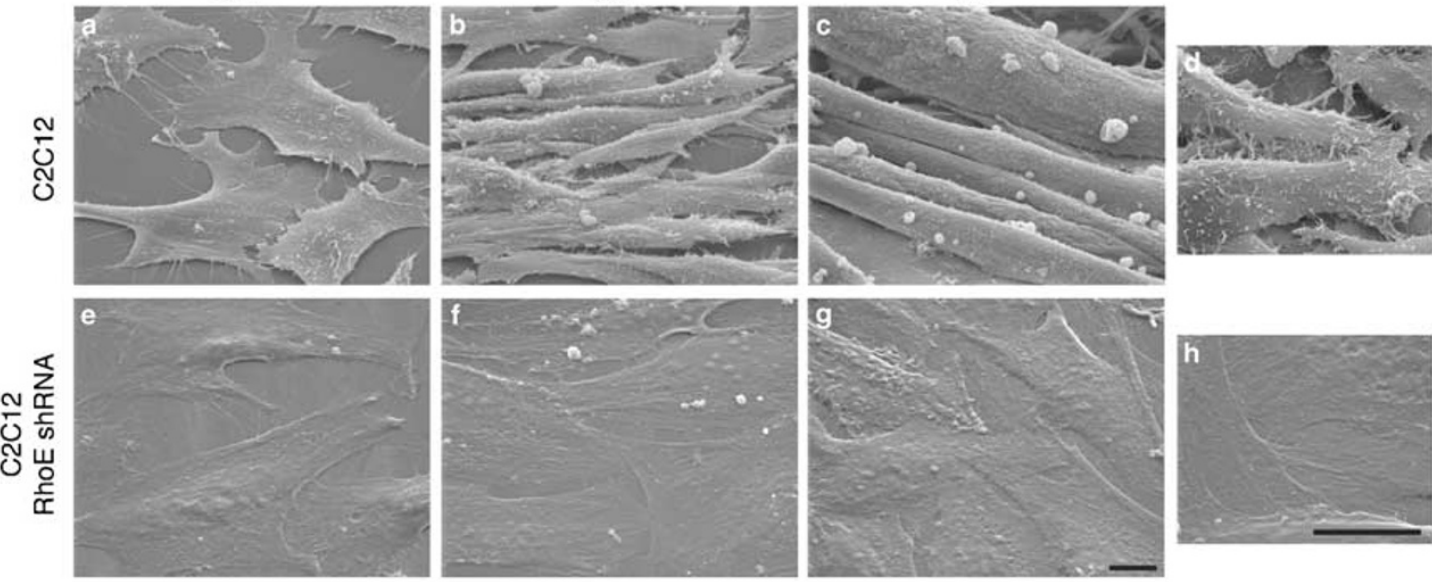

D a
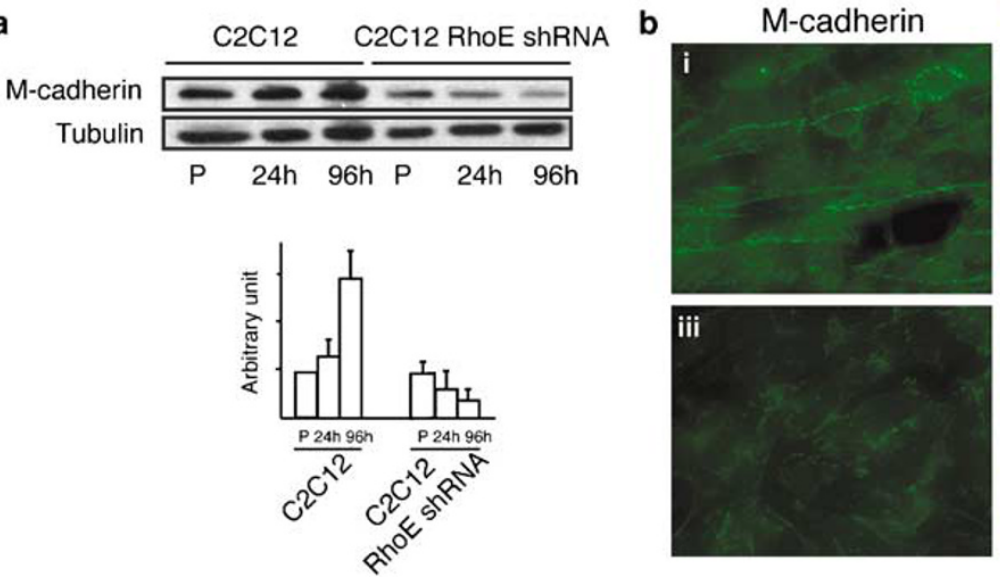

M-cadherin + Nuclei
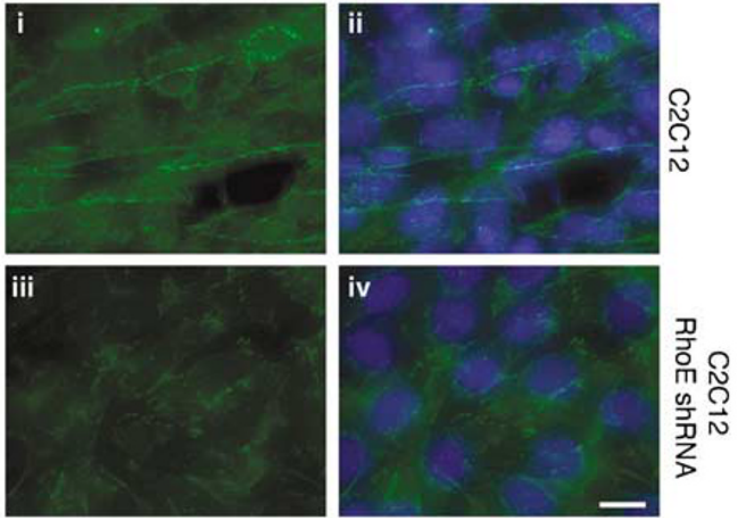

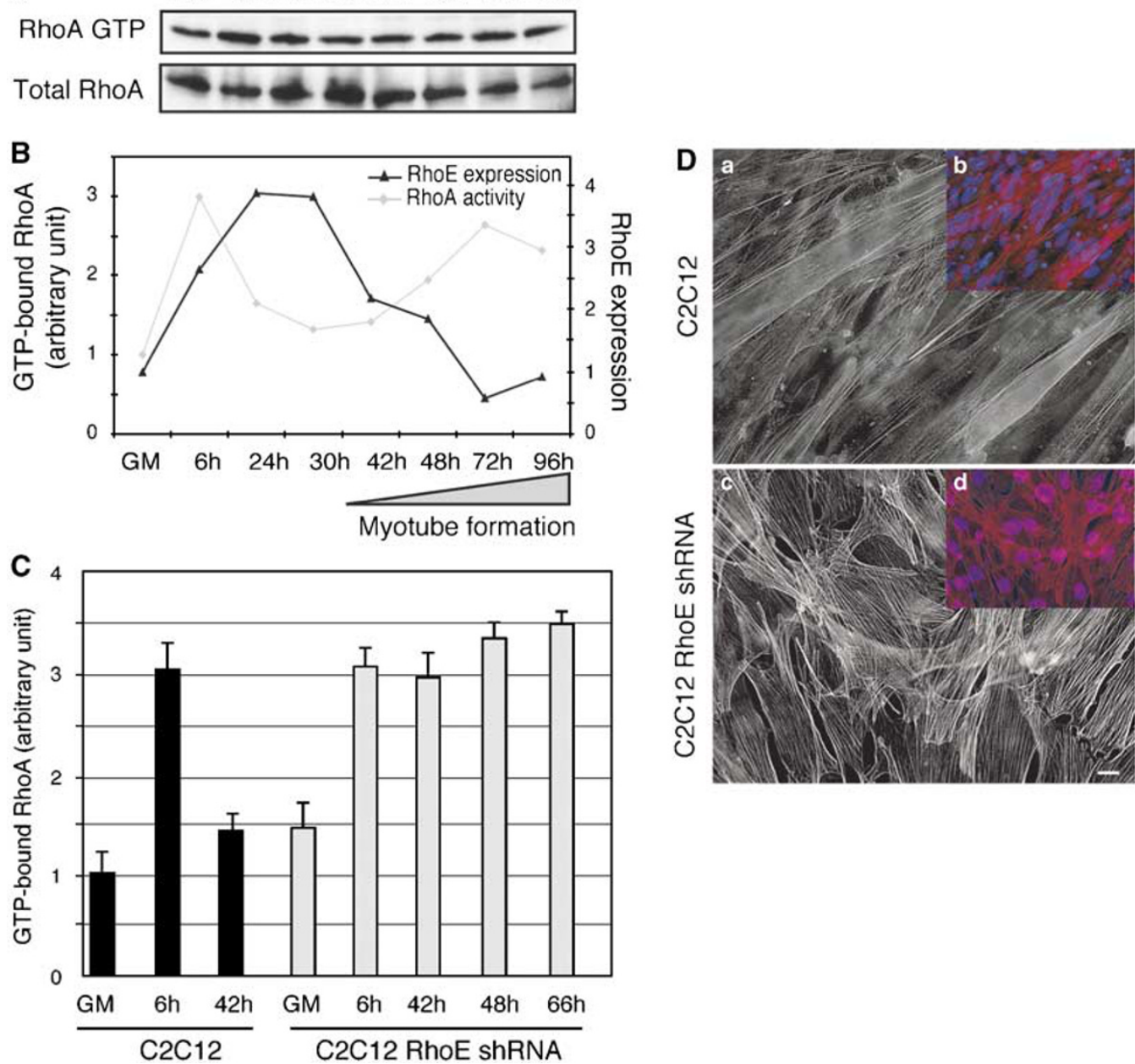

Figure 5 RhoE downregulates RhoA activity. (A) The level of GTP-bound RhoA was measured using GST fused to the Rho-binding domain of the RhoA effector Rhotekin (GST-RBD) in lysates obtained from C2C12 cells in GM or DM from the same kinetic as the one described in Figure 2. RhoA was detected by immunoblotting. Data are representative of three independent experiments. (B) RhoA GTPase activity normalized to the amount of total protein was calculated from panel $A$ and plotted with the RhoE protein levels in cell extracts from the same kinetic (shown in Figure 2B). (C) The level of GTP-bound RhoA was measured in lysates obtained from parental and C2C12 RhoE shRNA myoblasts that were in GM or DM and collected at the indicated times. Data are representative of three independent experiments. (D) Parental and C2C12 RhoE shRNA myoblasts were fixed after 3 days in DM and stained for F-actin ( $a$ and $c$ ). Overlay of F-actin (red) and DNA staining (blue) are shown in panels $b$ and d. Bar: $10 \mu \mathrm{m}$

ones (Figure 6B). These data show that RhoE is responsible for the downregulation of RhoA, a crucial step in myoblast fusion.

RhoE activates p190RhoGAP at the onset of myoblast fusion. p190RhoGAP is a RhoE effector involved in RhoEinduced inactivation of RhoA in fibroblasts. ${ }^{20}$ We thus tested whether RhoE can also interact with p190RhoGAP in C2C12 myoblasts. Immunoprecipitation using an anti-RhoE antibody was performed in extracts from $\mathrm{C} 2 \mathrm{C} 12$ myoblasts in $\mathrm{GM}$ or after the indicated times in DM (Figure 7A). Endogenous p190RhoGAP co-immunoprecipitated with RhoE (Figure 8Aa), and this association increased at the onset of myoblast fusion (Figure 7Ab).

We then analyzed the GAP activity of p190RhoGAP at different time points during myoblast differentiation in parental and $\mathrm{C} 2 \mathrm{C} 12$ RhoE shRNA myoblasts (Figure 7B). Activated p190RhoGAP was pulled down using GST-
RhoAL63, which is locked in its GTP-bound state. ${ }^{29}$ Pulleddown samples were then immunoblotted with an antip190RhoGAP antibody. The activity of p190RhoGAP gradually increased in $\mathrm{C} 2 \mathrm{C} 12$ myoblasts after $\mathrm{DM}$ addition. In contrast, in C2C12 RhoE shRNA myoblasts, we did not observe any increase in the activity of p190RhoGAP. These data support the notion that p190RhoGAP interaction with RhoE allows the increase of its activity during myoblast differentiation.

RhoE effect on myoblast fusion is also dependent on ROCK. RhoE binds to the RhoA effector ROCKI and inhibits its activity. ${ }^{21}$ Moreover, inhibition of ROCK activity is required for myoblast fusion. ${ }^{9,18}$ We thus analyzed whether ROCK was involved in the inhibition of myoblasts fusion induced by RhoE silencing. We first tested whether ROCKI was associated with $\mathrm{RhoE}$ in $\mathrm{C} 2 \mathrm{C} 12$ myoblasts using immunoprecipitation (Figure $8 \mathrm{~A}$ ). ROCKI co-immunoprecipitated with 
A

C2C12 Luci ShRNA

C2C12 RhoE shRNA

C2C12 RhoE shRNA
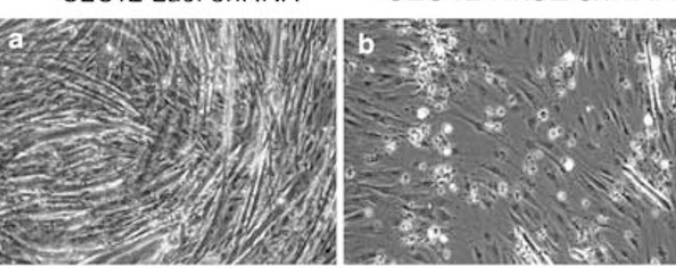

+ C3 transferase

B

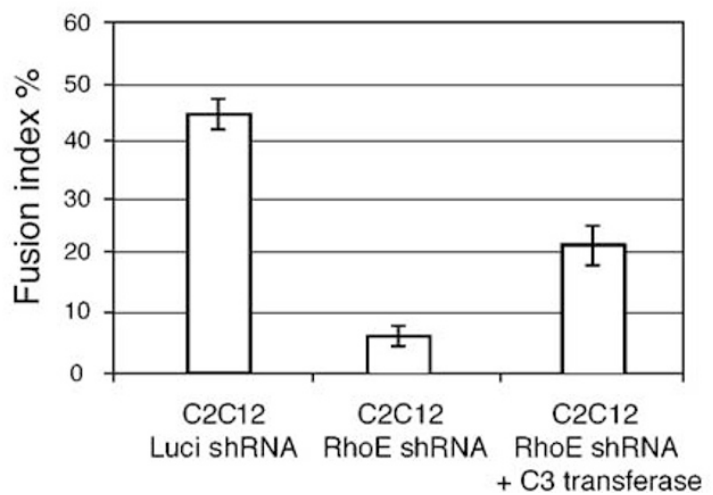

Figure 6 RhoA inhibition partially rescues myoblast fusion. Control C2C12 Luciand RhoE shRNA myoblasts were induced to differentiate by replacing the GM with the appropriate DM. Six hours later, C3 transferase was added, if appropriate. DM with or without C3 transferase was replaced every $24 \mathrm{~h}$. (A) Images of control C2C12 Luci shRNA (a) and C2C12 RhoE shRNA myoblasts (b) after 4 days in DM or C2C12 RhoE shRNA myoblasts after 4 days in DM containing C3 transferase (c). Bar, $50 \mu$ m. (B) The histogram represents the fusion index calculated $96 \mathrm{~h}$ after DM addition for control C2C12 Luci and RhoE shRNA C2C12 myoblasts treated, or not, with C3 transferase. Data are the mean \pm S.E.M. of three independent experiments, where at least 3000 nuclei/experiment were counted

RhoE in C2C12 myoblasts in GM or after the indicated times in DM. We also analyzed whether RhoE impacts on the binding of RhoA to ROCK1. We compared the association of RhoA to ROCK1 in parental and C2C12 RhoE shRNA expressing $\mathrm{C} 2 \mathrm{C} 12$ myoblasts. We found that the level of RhoA bound to ROCK increases upon RhoE silencing (Figure 8B). We next analyzed ROCK activity by assessing the phosphorylation of a ROCK substrate, the myosin binding subunit of the myosin light chain phosphatase MYPT- $1^{30}$ (Figure $8 \mathrm{C}$ ). As previously reported, we observed a decrease in ROCK activity during myogenesis in control cells, but not in C2C12 RhoE shRNA myoblasts induced to differentiate.

To gain insight into the role of ROCK in RhoE-induced myoblast fusion, we tested the effect of $\mathrm{Y}-27632$, a pharmacological ROCK inhibitor. C2C12 RhoE shRNA myoblasts were grown in DM in the presence of $\mathrm{Y}-27632$. Inhibition of ROCK partially rescued myoblast fusion in $\mathrm{C} 2 \mathrm{C} 12$ RhoE shRNA cells (Figure 8D), as confirmed by quantification of the fusion index (Figure 8E). These data argue for a participation of RhoE in ROCK inhibition required for myoblast fusion.

\section{Discussion}

Unraveling the molecular mechanisms that govern myoblast fusion is important for the understanding of muscle diseases where fusion is affected or for the repair process that involves adult satellite-cell fusion. Among the signaling pathways involved in myoblast fusion, the decrease in RhoA/ROCK activities appears to be crucial. ${ }^{8,9,18}$ We have searched for upstream regulators of RhoA/ROCK signaling during myoblast fusion and demonstrate here that RhoE plays an important role in their regulation and in skeletal muscle cell differentiation.

RhoE is required for myoblast fusion. Our results prove that $\mathrm{RhoE}$ is required for myoblast fusion in $\mathrm{C} 2 \mathrm{C} 12$ myoblasts, although it is dispensable for myogenesis induction. Indeed, RhoE knockdown impairs myotube formation without altering the level of muscle-specific gene products. We report modifications in RhoE expression during myogenesis consistent with a role during myoblast fusion, as its expression level in $\mathrm{C} 2 \mathrm{C} 12$ myoblasts increases until the onset of myoblast fusion before resuming its basal level once fusion is well under way. Its implication in muscle differentiation is reinforced by the results we obtained in an in vivo model for muscle regeneration. Moreover, the data of RhoE localization during myogenesis combined with the analysis of the phenotype of C2C12 myoblast, in which RhoE was silenced, suggest that RhoE participates in myoblast elongation and alignment before fusion. The variations in RhoE expression during myogenesis are consistent with the way this molecule is regulated. Indeed, RhoE binds only weakly to GDP, has very low, if any, intrinsic GTPase activity and therefore exists predominantly in a GTP-bound state. RhoE is not regulated by GEFs or GAPs but rather by its expression level and post-translational modifications such as phosphorylation. ${ }^{21,31}$ Further studies will be required to understand how RhoE expression is controlled during myogenesis. 
A a

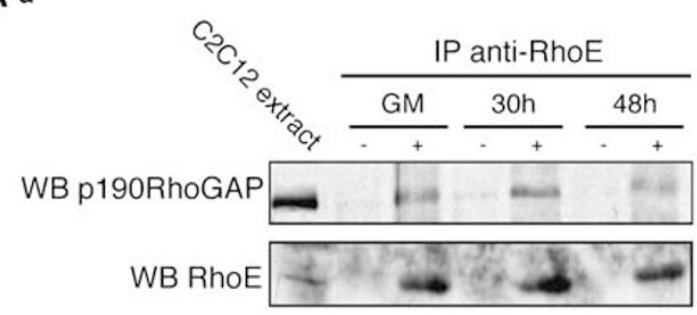

b

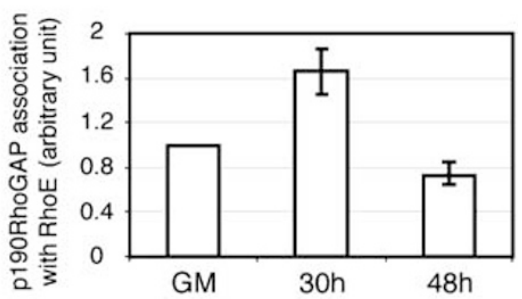

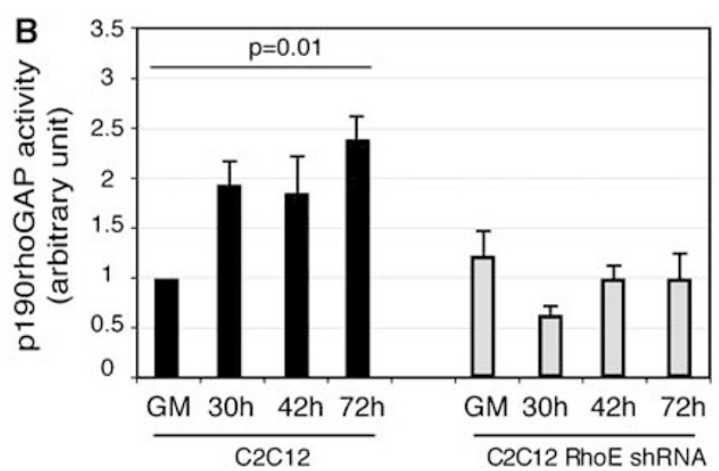

Figure 7 RhoE binds to p190RhoGAP and regulates its activity at the onset of the fusion process. (A) (a) Cell lysates of C2C12 myoblasts cultured in GM or at the indicated times after DM addition were immunoprecipitated using an anti-RhoE antibody and immunoblotted for the presence of p190RhoGAP and RhoE. -, without antiRhoE antibody; +, with anti-RhoE antibody. (b) The histogram shows the quantification of p190RhoGAP associated with RhoE. Data are the mean \pm S.E.M. of three independent experiments. (B) Control and RhoE shRNA C2C12 myoblasts cultured in GM or at different times after DM were lysed and subjected to pulldown analysis with GST-RhoAL63. The precipitated material and total lysates were analyzed for p190RhoGAP levels by immunoblotting. Three independent experiments were analyzed by densitometry. The histogram represents the GAP activity normalized to the total protein content

RhoE decreases RhoA and ROCK activities. RhoA GTPase activity is tightly controlled throughout myogenesis. At the beginning of myogenesis, RhoA is activated through an N-cadherin-mediated pathway ${ }^{10,27}$ and is then downregulated at the onset of myoblast fusion. ${ }^{8,18}$ We report here that RhoE is required for the decrease of RhoA activity during myoblast fusion. This is the first example of a regulatory pathway that negatively controls RhoA activity to allow fusion to occur. In fibroblasts, RhoE interacts with p190RhoGAP in vitro and increases its GAP activity toward GTP-bound RhoA. ${ }^{20}$ We demonstrate here, on endogenous proteins, that the highest association between RhoE and p190RhoGAP occurs at the onset of myoblast fusion. Interestingly, $\mathrm{C} 2 \mathrm{C} 12$ myoblasts in which RhoE was silenced show defects both in p190RhoGAP activation and RhoA inhibition at the onset of myoblast fusion. Finally, myoblast fusion could be partially rescued in $\mathrm{C} 2 \mathrm{C} 12$ RhoE shRNA by inactivating RhoA with $\mathrm{C} 3$ exoenzyme. In conclusion, these data explain how RhoE, through the binding and activation of its effector p190RhoGAP, antagonizes RhoA activity and participates in the induction of myoblast fusion. RhoE also inhibits RhoA signaling by binding to the RhoA-activated serine/threonine kinase ROCK, whose activity must be decreased during myogenic differentiation to allow myoblast fusion. ${ }^{9}$ Addition of the ROCK inhibitor Y-27632 partially rescued myoblast fusion in C2C12 RhoE shRNA myoblasts as efficiently as C3 the exoenzyme. Moreover, Y-27632 did not strengthen the effect of C3 exoenzyme alone (data not shown), suggesting that RhoA acts through ROCK to repress myoblast fusion.
Nevertheless, as the recovery of myoblast fusion with these drugs was only partial, other yet unknown RhoE effectors might participate in regulating myoblast fusion. For instance, the RhoE effector Socius is involved in RhoE-induced disassembly of actin stress fibers, ${ }^{32}$ and Rnd2 and RhoE interact with the RhoGAP MgcRacGAP. ${ }^{33}$ Nevertheless, both Socius and MgcRacGAP are predominantly expressed in the testis, which emphasized the need to identify other skeletal muscle-specific RhoE effectors.

Spatial RhoE targeting. The mechanisms responsible for RhoE activation and function in myoblast fusion are most probably linked to its increased expression, which allows its accumulation above a threshold level, thus favoring its binding to p190RhoGAP and ROCK1 that leads to the inhibition of the RhoA/ROCK pathway. Moreover, RhoE recruitment to specific plasma membrane sites might facilitate its association with p190RhoGAP and ROCK1. Indeed, in fibroblasts and epithelial cells, RhoE colocalizes with cadherins in adherens junctions. ${ }^{34}$ Although M-cadherin is involved in myoblast fusion, ${ }^{8}$ we could not detect any association of RhoE with either $\mathrm{M}$-cadherin or $\mathrm{N}$-cadherin in C2C12 myoblasts (data not shown). Also $\beta 1$-integrin, another transmembrane protein involved in myoblast fusion, was not associated with RhoE (data not shown).$^{35}$ In neurons, RhoE and Rnd1 interact with plexins, which are semaphorin receptors involved in axon guidance through regulation of p190RhoGAP-dependent RhoA downregulation. ${ }^{28,36}$ Semaphorin $4 \mathrm{C}$ participates in myoblast fusion, ${ }^{37}$ and 
A

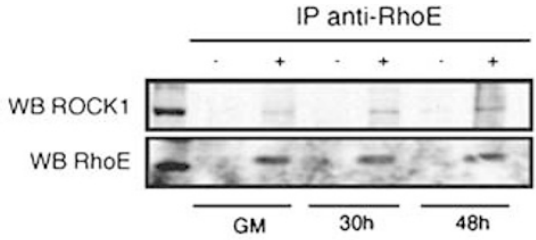

B

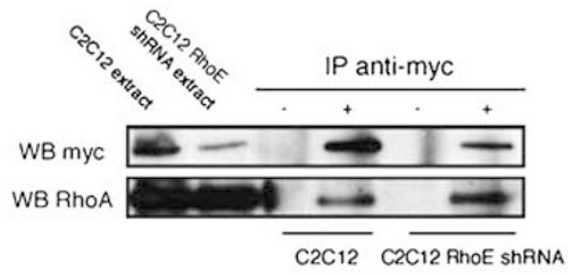

C

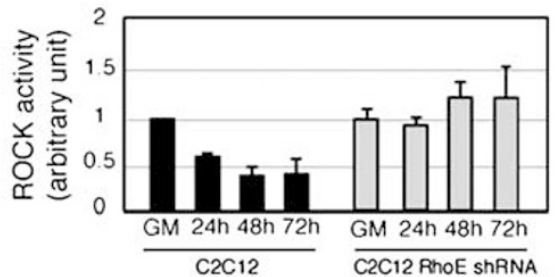

D

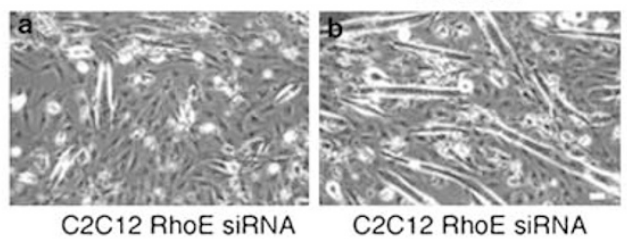

E

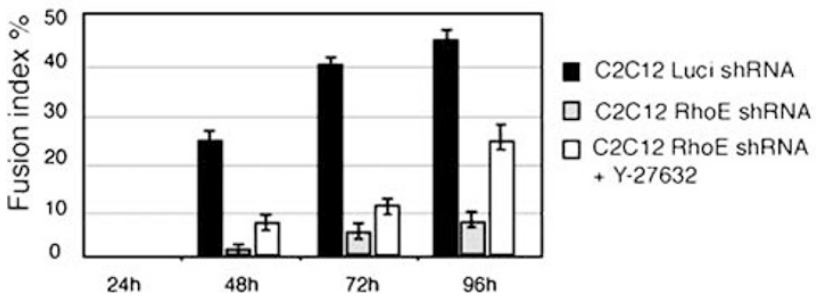

Figure 8 ROCK inhibition partially rescues myoblast fusion in RhoE deficient cells. (A) Cell lysates of $\mathrm{C} 2 \mathrm{C} 12$ myoblasts cultured in $\mathrm{GM}$ or at different times after DM addition were immunoprecipitated using an anti-RhoE antibody and immunoblotted to assess the presence of ROCK1 and RhoE. - , without antiRhoE antibody; +, with anti-RhoE antibody. (B) Parental and RhoE shRNA expressing $\mathrm{C} 2 \mathrm{C} 12$ myoblasts cultured in GM were co-transfected with GFP-tagged RhoA and myc-tagged ROCK1 expressing plasmids. Cell lysates were immunoprecipitated using a mouse monoclonal anti-myc antibody and immunoblotted to assess the presence of ROCK1 (WB myc) and RhoA (WB RhoA). without anti-myc antibody; + , with anti-myc antibody. (C) Phospho-MYPT-1 and $\alpha-$ Tubulin levels were analyzed in total lysates by immunoblotting from control and C2C12 RhoE shRNA myoblasts cultured in GM or at different times after DM addition. The histogram shows the quantification of MYPT-1 phosphorylation normalized to the total protein content. Data are the mean \pm S.E.M. of three independent experiments. (D) Images of $\mathrm{C} 2 \mathrm{C} 12$ RhoE shRNA myoblasts after 4 days in DM (a) or DM containing Y-27632 (b). Bar, $50 \mu \mathrm{m}$. (E) The histogram represents the fusion index calculated at the indicated times after DM addition for control C2C12 Luci and RhoE shRNA myoblasts treated or not with Y-27632. Data are the mean \pm S.E.M. of three independent experiments, where at least 3000 nuclei/experiments were counted therefore it would be important to test whether RhoE associates with this kind of complex in myoblasts.

RhoE/RhoA/ROCKI signaling and myoblast fusion. Here we demonstrate for the first time that RhoE participates in skeletal muscle differentiation through the downregulation of RhoA/ROCK signaling, an event required for myoblast fusion. ${ }^{8,9}$ ROCK phosphorylates both the myosin binding subunit of myosin light chain phosphatase and the myosin regulatory light chain, which induces the actin-myosin 2 interaction and promotes actin cytoskeleton assembly and cell contractility. ${ }^{30,38}$ When RhoA and ROCK activities are decreased, Myosin 2 activity is also reduced. In $\mathrm{C} 2 \mathrm{C} 12$ myoblasts in which $R h o E$ was silenced, stress fibers are not reduced and myoblast fusion is impaired. Interestingly, the loss of stress fibers occurs in pre-fusing myoblasts. ${ }^{25}$ Nevertheless, we could only partially rescue myoblast fusion with RhoA or ROCK inhibitors, and synergy was not obtained by the addition of both (data not shown). Although we cannot rule out a possible toxicity of these drugs, this suggests the existence of other RhoE-dependent pathways controlling myoblast fusion. Indeed, we found that the inhibitor of myosin light chain kinase (MLCK) ML7 slightly increases the effect of $\mathrm{C} 3$ exoenzyme on the recovery of myoblast fusion in RhoE-deficient myoblasts (data not shown). Myosin 2 inhibition with blebbistatin in $\mathrm{C} 2 \mathrm{C} 12$ myoblasts in which RhoE was silenced also resulted in rescue of myoblast fusion (data not shown), supporting the idea that a decrease in acto-myosin contraction is required for myoblast fusion. These data suggest a synergy between a RhoA/ROCK and a MLCK pathway downstream of RhoE, both converging on the reduction of myosin activity. Upstream regulation of MLCK can occur through Rac1 and its effector Pak, which phosphorylates and inactivates MLCK. Moreover, there is a functional antagonistic balance between the Rho and Rac subgroups of the Rho family, and we have previously reported such a crosstalk in skeletal muscle cells. $^{8,17}$ Indeed, Rac1 signaling is required for myoblast fusion, both in Drosophila and in mammalian myoblasts. ${ }^{4-7,17}$ Interestingly, our detailed examination of the membrane morphological modifications by SEM and pulldown assay (data not shown) indicate that Rac1 activity is not increased in $\mathrm{C} 2 \mathrm{C} 12$ myoblasts in which $R h o E$ was knocked down. Thus RhoE-dependent reduction of RhoA activity might also contribute to Rac1 activation. Our results establish that RhoE-dependent downregulation of RhoA/ROCK signaling also contributes to correct functioning of $\mathrm{M}$ cadherin, a protein required for myoblast fusion, which is regulated by RhoA GTPase. ${ }^{8}$ Indeed, we show that $M$ cadherin is downregulated and mislocalized in myoblast in which $R h o E$ was silenced and that its expression is partially rescued by the addition of RhoA or ROCK inhibitors (data not shown).

Myoblast fusion is a multi-step process in which actin cytoskeleton dynamics plays a key role. We thus propose that RhoE is an actor of myoblast fusion through the regulation of the organization of the actin cytoskeleton and M-cadherin function, which in turn controls myoblast alignment and elongation. 


\section{Materials and Methods}

Cell culture. $\mathrm{C} 2 \mathrm{C} 12$ mouse myoblasts were cultured as described. ${ }^{27}$ To induce differentiation, GM was replaced by DM consisting of DMEM/Ham's F-12 supplemented with $2 \%$ FCS. Stable cell lines derived from C2C12 myoblasts were cultured under the same conditions. Cell permeable C3 transferase $(0.4 \mu \mathrm{g} / \mathrm{ml}$, Cytoskeleton) or the ROCK inhibitor Y-27632 (10 $\mu \mathrm{M}$, Calbiochem) were added $6 \mathrm{~h}$ after the addition of DM. Drug-containing DM was refreshed every $24 \mathrm{~h}$.

Quantification of mRNA by real-time quantitative PCR amplification. Total RNAs from myoblasts in culture were prepared using the Rneasy minikit from QIAGEN. Total RNAs from TA muscles were extracted following the acid/guanidinium thiocyanate/phenol/chloroform method using RNable (Eurobio, France) according to the manufacturer's instructions. cDNAs were synthetized from $5 \mathrm{mg}$ of total RNA using superscript II Reverse Transcriptase (Invitrogen) and random hexamer primers. The levels of the various CDNAs were determined by quantitative PCR amplification using the SYBR green I with a Light Cycler (Roche Diagnostics, Sommerville, NJ, USA). All primers used were previously described. ${ }^{22,39}$ GAPDH mRNA was amplified as an internal control. The specificity of the primers was checked by DNA sequencing.

Gel electrophoresis and immunoblotting. Cell extracts were prepared as previously described. ${ }^{8}$ Protein concentration was determined with a BCA protein assay kit (Pierce Chemical, Rockford, IL, USA). Protein extracts $(20 \mu \mathrm{g})$ were resolved on polyacrylamide gels $(12 \%)$ and transferred onto Immobilon-P membranes. Membranes were incubated with monoclonal antibodies against RhoE (1:1000; Upstate), troponin T (1:1000; Sigma-Aldrich), myogenin (1:500; BD Biosciences PharMingen, San Diego, CA, USA), M-cadherin (1:200, NanoTools, Munich, Germany) and $\alpha$-tubulin (1:100). Membranes were processed as described previously. ${ }^{8}$

Small hairpin RNA retroviral vector construction and establishment of stable cell lines. To suppress endogenous RhoE expression, annealed double strand oligonucleotides GATCCGTCAGAACGT GAAATGCAAAGTTCAAGAGACTTGCATTTCACGTTCTGACTTTTTTACGCGTG (top) and AATTCACGCGTAAAAAAGTCAGAACGTGAAATGCAAGTCTCTTGA ACTTTGCATTTCACGTTCTGACG (bottom) were inserted into the RNAi-Ready pSIREN-RetroQ-ZsGreen vector (Clontech, BD Biosciences). Bold letters correspond to oligonucleotides $291-310$ of the mouse and human RhoE cDNA sequence (NM_028810). The PSIREN-RetroQ-ZsGreen vector, which targets the firefly Luciferase, was used as a control.

Phoenix cells expressing RhoE shRNA or Luciferase shRNA were grown to collect retrovirus-containing cell-free supernatants. Infection was performed on exponentially growing $\mathrm{C} 2 \mathrm{C} 12$ myoblasts $\left(5 \times 10^{5}\right.$ cells per 60 -mm dish) by using $5 \mathrm{ml}$ of viral supernatant. Positive GFP cells were selected by FACS and cloned by limited dilution. RhoE expression was assayed on five random clones for RhoE shRNA and on a pool of Luciferase shRNA expressing $\mathrm{C} 2 \mathrm{C} 12$.

Fusion index determination. Cells growing onto 100-mm dishes were fixed in $3.7 \%$ formaldehyde in phosphate-buffered saline (PBS) followed by a 3-min permeabilization in $0.1 \%$ Triton X-100 in PBS and saturated in PBS containing $0.1 \%$ bovine serum albumin. Nuclei were stained with Hoechst stain $(0.1 \mu \mathrm{g} / \mathrm{ml}$, SigmaAldrich) and washed with PBS. Fusion index, that is the number of nuclei in multinucleated myotubes divided by the total number of nuclei, was determined by the MRI Cell Image Analyzer software. ${ }^{8}$ At least 7000 nuclei in each experiment were counted. Only cells with three nuclei or more were considered to be myotubes.

May-Grünwald Giemsa staining. Cells were washed twice with PBS, incubated with May-Grünwald diluted in PBS for 5 min and next with Giemsa diluted in PBS for $10 \mathrm{~min}$.

Muscle regeneration model. Mice (male C57BL/6J, 6 week old) were anesthetized by intraperitoneal injection of pentobarbital. Twenty microliters of $1 \mu \mathrm{M}$ notexin (Latoxan, Valence, France) or $20 \mu \mathrm{l}$ of $10 \mu \mathrm{M}$ cardiotoxin (Latoxan, Valence, France) were injected into the TA muscles. Muscles were collected at various times after injection. Contralateral uninjected TA muscles were used as control. Muscles were used for hematoxylin/eosin staining and to isolate total RNA for RT-PCR.

Immunocytochemistry. Cells were fixed in $4 \%$ paraformaldehyde in PBS followed by a 2-min permeabilization in $0.1 \%$ Triton X-100 (in PBS). RhoE was stained using an affinity-purified antibody raised against an N-terminal peptide of RhoE (SQKLSSKSIMDPNQNVK) as previously described, ${ }^{21}$ and produced by Eurogentec (Seraing, Belgium). Control experiments were performed using the preimmune serum and affinity-purified anti-RhoE blocked with saturating levels of its specific peptide antigen. M-cadherin was stained using a polyclonal antibody as described. $^{8}$ Anti-RhoE and anti-M-cadherin were revealed using Alexa Fluor 488conjugated goat anti-rabbit antibody (Molecular Probes, Invitrogen). F-actin expression was analyzed using TRITC-conjugated phalloidin (Sigma-Aldrich) and nuclei were stained with Hoechst ( $0.1 \mu \mathrm{g} / \mathrm{ml}$, Sigma-Aldrich). Images were captured with a MicroMax 1300 CCD camera (RS-Princeton Instruments, Treuton, NJ, USA) driven by the MetaMorph (v.7, Universal Imaging Corp, Westchester, PA, USA) Software. Stacks of images were captured with a piezo stepper (E662, Physik Instruments) with a Z step of $0.2 \mu \mathrm{m}$. Stacks were then restored with Huygens deconvolution software (Scientific Volume Imaging) and the restored images were viewed in 3D with MetaMorph. Images were processed using Adobe Photoshop and Adobe Illustrator.

Scanning electron microscopy. Parental and C2C12 RhoE shRNA myoblasts were grown on Thermanox ${ }^{\mathrm{TM}}$ coverslips (Nunc) either in GM or in DM. After washing in PBS, myoblasts were fixed in PBS containing $3 \%$ glutaraldehyde for $2 \mathrm{~h}$ and post-fixed with $1 \% \mathrm{OsO}_{4}$ in distilled water for $1 \mathrm{~h}$. After alcohol dehydration, myoblasts were dried in hexamethyldisilazane (Acros Organics, $\mathrm{NJ}$, USA) for $2 \mathrm{~min}$, coated with Gold-palladium and observed using a Hitachi S4000 scanning microscope at $10 \mathrm{kV}$. For each condition, at least 100 cells were examined.

RhoA GTPase activity assay. Proliferating or differentiating $\mathrm{C} 2 \mathrm{C} 12$ myoblasts were lysed and processed to measure the total and GTP-bound RhoA levels as described previously ${ }^{8}$ or by using the luminometry based G-LISA ${ }^{\text {TM }}$ Rho activation assay (Cytoskeleton Inc.).

Immunoprecipitation. $\mathrm{C} 2 \mathrm{C} 12$ cells were lysed for $10 \mathrm{~min}$ in ice-cold extraction protein buffer. ${ }^{8}$ Protein extracts $(1 \mathrm{mg})$ were immunoprecipitated using a mouse monoclonal anti-RhoE antibody (1/1000 dilution; Upstate). Negative controls were performed using IgG from murine serum (Sigma-Aldrich). Half of each immunoprecipitation was separated on an $8 \%$ polyacrylamide gel and then transferred onto nitrocellulose. p190RhoGAP and ROCKI detection were performed using monoclonal antibody (1/250; BD Transduction Laboratories). The other half of each immunoprecipitation was separated on a $15 \%$ polyacrylamide gel, transferred on Immobilon-P membranes and immunoblotted with a polyclonal anti-RhoE antibody.

Three 100-mm dishes were co-transfected with $3 \mu \mathrm{g}$ GFP-tagged Rho and myc-tagged ROCK1 plasmids using JetPEl. Twenty-four hours after the transfection, cells were lysed and protein extracts $(2 \mathrm{mg})$ were immunoprecipitated using a mouse monoclonal anti-myc antibody from ascites (1/1000 dilution). Negative controls were performed using IgG from murine serum (Sigma-Aldrich). Eighty percent of each immunoprecipitation was separated on an $8 \%$ polyacrylamide gel and then transferred onto nitrocellulose. ROCKI detection was performed using monoclonal anti-myc antibody from ascites (1/10000 dilution).

Twenty percent of each immunoprecipitation was separated on a $15 \%$ polyacrylamide gel, transferred on Immobilon-P membranes and immunoblotted with a monoclonal anti-RhoA antibody (1/300 dilution, Santa Cruz, Tebu).

p190 RhoGAP activity assay. RhoA L63GST (a generous gift from $\mathrm{K}$ Burridge) was prepared as described. ${ }^{29}$ Cells were lysed at different times after DM addition and processed as described. ${ }^{28}$

ROCK activity assay. Cells extracts were lysed as described. ${ }^{40}$ Protein extracts $(60 \mu \mathrm{g})$ were resolved on polyacrylamide gels $(10 \%)$ and transferred onto Immobilon-P membranes. Membranes were probed with phospho-Mypt1 polyclonal antibody (1/1000) (Upstate) and $\alpha$-tubulin (1:100).

Acknowledgements. We thank Evelyne Bloch-Gallego, Philippe Fort, Pierre Roux and Nicolas Taulet for helpful discussions, and Keith Burridge for the RhoAL63GST expression vector. Electron scanning microscopy was performed at the Centre Regional d'Imagerie Cellulaire de Montpellier. This work was supported by the Association Française contre les Myopathies, the Agence Nationale de la Recherche (ANR-06-BLAN-0389), and the Association Française pour la 
Recherche contre le Cancer. CGR was supported by INSERM and JK by the Ligue Nationale contre le Cancer.

1. Jaffe AB, Hall A. Rho GTPases: biochemistry and biology. Annu Rev Cell Dev Biol 2005; 21: $247-269$

2. Carnac G, Primig M, Kitzmann M, Chafey $P$, Tuil D, Lamb $N$ et al. RhoA GTPase and serum response factor control selectively the expression of MyoD without affecting myf5 in mouse myoblasts [In Process Citation]. Mol Biol Cell 1998; 9: 1891-1902.

3. Meriane M, Roux P, Primig M, Fort P, Gauthier-Rouviere C. Critical activities of Rac1 and $\mathrm{Cdc42Hs}$ in skeletal myogenesis: antagonistic effects of JNK and p38 pathways. Mol Biol Cell 2000; 11: 2513-2528.

4. Luo L, Liao YJ, Jan LY, Jan YN. Distinct morphogenetic functions of similar small GTPases: Drosophila Drac1 is involved in axonal outgrowth and myoblast fusion. Genes Dev 1994; 8: 1787-1802

5. Hakeda-Suzuki S, Ng J, Tzu J, Dietzl G, Sun Y, Harms M et al. Rac function and regulation during Drosophila development. Nature 2002; 416: 438-442.

6. Fernandes JJ, Atreya KB, Desai KM, Hall RE, Patel MD, Desai AA et al. A dominant negative form of Rac1 affects myogenesis of adult thoracic muscles in Drosophila. Dev Bio 2005; 285: 11-27.

7. Erickson MR, Galletta BJ, Abmayr SM. Drosophila myoblast city encodes a conserved protein that is essential for myoblast fusion, dorsal closure, and cytoskeletal organization J Cell Biol 1997; 138: 589-603.

8. Charrasse S, Comunale F, Grumbach Y, Poulat F, Blangy A, Gauthier-Rouviere C. RhoA GTPase regulates M-cadherin activity and myoblast fusion. Mol Biol Cell 2006; 17: 749-759.

9. Castellani L, Salvati E, Alema S, Falcone G. Fine regulation of RhoA and Rock is required for skeletal muscle differentiation. J Biol Chem 2006; 281: 15249-15257.

10. Lovett FA, Gonzalez I, Salih DA, Cobb LJ, Tripathi G, Cosgrove RA et al. Convergence of Igf2 expression and adhesion signalling via RhoA and p38 MAPK enhances myogenic differentiation. J Cell Sci 2006; 119 (Part 23): 4828-4840.

11. Foster R, Hu KQ, Lu Y, Nolan KM, Thissen J, Settleman J. Identification of a novel human Rho protein with unusual properties: GTPase deficiency and in vivo farnesylation. $\mathrm{Mol} \mathrm{Ce}$ Biol 1996; 16: 2689-2699.

12. Guasch RM, Scambler P, Jones GE, Ridley AJ. RhoE regulates actin cytoskeleton organization and cell migration. Mol Cell Biol 1998; 18: 4761-4771.

13. Nobes CD, Lauritzen I, Mattei MG, Paris S, Hall A, Chardin P. A new member of the Rho family, Rnd1, promotes disassembly of actin filament structures and loss of cell adhesion. J Cell Biol 1998; 141: 187-197.

14. Chardin P. Function and regulation of Rnd proteins. Nat Rev Mol Cell Biol 2006; 7: 54-62.

15. Meriane M, Charrasse S, Comunale F, Mery A, Fort $P$, Roux $P$ et al. Participation of small GTPases Rac1 and Cdc42Hs in myoblast transformation. Oncogene 2002; 21: 2901-2907.

16. Nolan KM, Barrett K, Lu Y, Hu KQ, Vincent S, Settleman J. Myoblast city, the Drosophila homolog of DOCK180/CED-5, is required in a Rac signaling pathway utilized for multiple developmental processes. Genes Dev 1998; 12: 3337-3342.

17. Charrasse S, Comunale F, Fortier M, Portales-Casamar E, Debant A, Gauthier-Rouviere C. M-cadherin activates Rac1 GTPase through the Rho-GEF trio during myoblast fusion. Mol Biol Cell 2007; 18: 1734-1743.

18. Nishiyama T, Kii I, Kudo A. Inactivation of Rho/ROCK signaling is crucial for the nuclea accumulation of FKHR and myoblast fusion. J Biol Chem 2004; 279: 47311-47319.

19. Chardin P. GTPase regulation: getting aRnd Rock and Rho inhibition. Curr Biol 2003; 13 R702-R704.

20. Wennerberg K, Forget MA, Ellerbroek SM, Arthur WT, Burridge K, Settleman J et al. Rnd proteins function as RhoA antagonists by activating p190 RhoGAP. Curr Biol 2003; 13 $1106-1115$.
21. Riento K, Guasch RM, Garg R, Jin B, Ridley AJ. RhoE binds to ROCK I and inhibits downstream signaling. Mol Cell Biol 2003; 23: 4219-4229.

22. Brazier H, Stephens S, Ory S, Fort P, Morrison N, Blangy A. Expression profile of RhoGTPases and RhoGEFs during RANKL-stimulated osteoclastogenesis: identification of essential genes in osteoclasts. J Bone Miner Res 2006; 21: 1387-1398.

23. Riento K, Totty N, Villalonga P, Garg R, Guasch R, Ridley AJ. RhoE function is regulated by ROCK I-mediated phosphorylation. EMBO J 2005; 24: 1170-1180.

24. Burattini S, Ferri P, Battistelli M, Curci R, Luchetti F, Falcieri E. C2C12 murine myoblasts as a model of skeletal muscle development: morpho-functional characterization. Eur $J$ Histochem 2004; 48: 223-233.

25. Swailes NT, Knight PJ, Peckham M. Actin filament organization in aligned prefusion myoblasts. J Anat 2004; 205: 381-391.

26. Kaufmann U, Kirsch J, Irintchev A, Wernig A, Starzinski-Powitz A. The M-cadherin catenin complex interacts with microtubules in skeletal muscle cells: implications for the fusion of myoblasts. J Cell Sci 1999; 112 (Part 1): 55-68.

27. Charrasse S, Meriane M, Comunale F, Blangy A, Gauthier-Rouviere C. N-cadherindependent cell-cell contact regulates Rho GTPases and beta-catenin localization in mouse C2C12 myoblasts. J Cell Biol 2002; 158: 953-965.

28. Barberis D, Casazza A, Sordella R, Corso S, Artigiani S, Settleman J et al. p190 Rho-GTPase activating protein associates with plexins and it is required for semaphorin signalling. J Cell Sci 2005; 118 (Part 20): 4689-4700.

29. Garcia-Mata R, Wennerberg K, Arthur WT, Noren NK, Ellerbroek SM, Burridge K Analysis of activated GAPs and GEFs in cell lysates. Methods Enzymol 2006; 406: 425-437.

30. Kimura K, Ito M, Amano M, Chihara K, Fukata $Y$, Nakafuku M et al. Regulation of myosin phosphatase by Rho and Rho-associated kinase (Rho-kinase). Science 1996; 273: 245-248.

31. Hansen SH, Zegers MM, Woodrow M, Rodriguez-Viciana P, Chardin P, Mostov KE et al. Induced expression of Rnd3 is associated with transformation of polarized epithelial cells by the Raf-MEK-extracellular signal-regulated kinase pathway. Mol Cell Biol 2000; 20: 9364-9375

32. Katoh $\mathrm{H}$, Harada A, Mori $\mathrm{K}$, Negishi M. Socius is a novel Rnd GTPase-interacting protein involved in disassembly of actin stress fibers. Mol Cell Biol 2002; 22: 2952-2964.

33. Naud N, Toure A, Liu J, Pineau C, Morin L, Dorseuil O et al. Rho family GTPase Rnd2 interacts and co-localizes with MgcRacGAP in male germ cells. Biochem $\mathrm{J}$ 2003; 372 (Part 1): 105-112.

34. Rubenstein NM, Chan JF, Kim JY, Hansen SH, Firestone GL. Rnd3/RhoE induces tight junction formation in mammary epithelial tumor cells. Exp Cell Res 2005; 305: 74-82.

35. Schwander M, Leu M, Stumm M, Dorchies OM, Ruegg UT, Schittny J et al. Beta1 integrins regulate myoblast fusion and sarcomere assembly. Dev Cell 2003; 4: 673-685

36. Oinuma I, Katoh H, Harada A, Negishi M. Direct interaction of Rnd1 with Plexin-B1 regulates PDZ-RhoGEF-mediated Rho activation by Plexin-B1 and induces cell contraction in COS-7 cells. J Biol Chem 2003; 278: 25671-25677.

37. Ko JA, Gondo T, Inagaki S, Inui M. Requirement of the transmembrane semaphorin Sema4C for myogenic differentiation. FEBS Lett 2005; 579: 2236-2242.

38. Katoh K, Kano Y, Amano M, Onishi H, Kaibuchi K, Fujiwara K. Rho-kinase-mediated contraction of isolated stress fibers. J Cell Biol 2001; 153: 569-584.

39. Charrasse S, Comunale F, Gilbert E, Delattre O, Gauthier-Rouviere C. Variation in cadherins and catenins expression is linked to both proliferation and transformation of Rhabdomyosarcoma. Oncogene 2004; 23: 2420-2430.

40. Wu Y, Erdodi F, Muranyi A, Nullmeyer KD, Lynch RM, Hartshorne DJ. Myosin phosphatase and myosin phosphorylation in differentiating C2C12 cells. J Muscle Res Cell Motil 2003; 24: $499-511$. 